\title{
Can Anti-Tumor Necrosis Factor Agents Be Discontinued in Patients with Inflammatory Bowel Disease?
}

\author{
Jihye Park ${ }^{1,2}$ and Jae Hee Cheon ${ }^{1,2,3}$ \\ ${ }^{1}$ Department of Internal Medicine, ${ }^{2}$ Institute of Gastroenterology, and ${ }^{3}$ Brain Korea 21 PLUS Project for Medical Science, Yonsei \\ University College of Medicine, Seoul, Korea
}

\author{
Corresponding Author \\ Jae Hee Cheon \\ ORCID https://orcid.org/0000-0002-2282-8904 \\ E-mail Geniushee@yuhs.ac
}

\begin{abstract}
See "Long-term Outcomes after the Discontinuation of Anti-Tumor Necrosis Factor-a Therapy in Patients with Inflammatory Bowel Disease under Clinical Remission: A Korean Association for the Study of Intestinal Disease Multicenter Study" by Joo Hye Song, et al. on page 752 , Vol. 15, No. 5, 2021
\end{abstract}

Inflammatory bowel diseases (IBD), including ulcerative colitis (UC) and Crohn's colitis (CD), are characterized by chronic and recurrent inflammation of the gastrointestinal tract. Anti-tumor necrosis factor (TNF) agents proved to be effective and safe in IBD. ${ }^{1,2}$ However, there are still concerns about the risk of malignancy and infections after long-term use of anti-TNF agents. ${ }^{3,4}$ There have been few data regarding the discontinuation of anti-TNF agents in IBD patients who achieved remission. This paper presents a study that focuses on the discontinuation of antiTNF agents and evaluates risk factors in predicting relapse of the disease.

In the current issue, Song et al. ${ }^{5}$ conducted a retrospective, multicenter, uncontrolled study. They analyzed a total of $109 \mathrm{IBD}$ patients $(71 \mathrm{CD}$ and $38 \mathrm{CD}$ ) treated with a first line anti-TNF agent (93 infliximab, 15 adalimumab, or one golimumab), and the median duration of the anti-TNF agent treatment was 12 months (range, 9.0 to 26.5 months). The cumulative relapse rates at $1,2,3$, and 5 years for IBD patients were $17.4 \%, 32.9 \%, 46.7 \%$, and $62.9 \%$, respectively. The cumulative relapse rates for $\mathrm{CD}$ patients were $11.3 \%$, $31.4 \%, 46.7 \%$, and $62.5 \%$, and those for UC patients, were $28.9 \%, 34.8 \%, 45.3 \%$, and $60.9 \%$, respectively. ${ }^{5}$ They suggested discontinuation owing to patients' preference (vs clinician's decision) and adalimumab group (vs infliximab group) as risk factors of disease relapse in $\mathrm{CD}$ patients. The non-mucosal healing group (vs mucosal healing group) in UC was suggested to be a high-risk group of disease relapse. Despite its retrospective design, lack of therapeutic drug monitoring, and inadequate measurement of endoscopic parameters, this study appears to be meaningful because it is the first report about the discontinuation of antiTNF agents in the real-life clinical practice in Korea.

Recently, Kobayashi et al. ${ }^{6}$ performed a first multicenter, open-labeled randomized controlled trial (RCT) in patients with UC treated with first line infliximab, and the median duration of infliximab treatment in discontinued infliximab group was 164.6 weeks. The median disease duration of discontinued infliximab group was 5.4 years. The cumulative relapse rates at $8,16,24,32,40$, and 48 weeks for UC patients were $6.5 \%, 23.9 \%, 26.1 \%, 32.6 \%$, $34.8 \%$, and $45.7 \%$, respectively. The relapse rates of UC in the study by Song et al. was relatively lower, approximately $20 \%$, compared to this RCT. The study population of in the study by Song et al. had a higher rate of ulcerative proctitis compared to the study by Kobayashi et al. (23.7\% vs $2.0 \%$ ). Also, the maintenance duration of anti-TNF in the study by Song et al. was relatively shorter compared to the study by Kobayashi et al. (48 weeks vs 164.6 weeks). The reasons for discontinuation of anti-TNF agents were various in real-life settings, which includes not only clinician's decision but also patient's preference. The relatively shorter maintenance duration of anti-TNF may indicate a lower inflammatory disease burden, which might have affected the outcomes.

In terms of $\mathrm{CD}$, Louis et al. ${ }^{7}$ performed a prospective, multicenter, uncontrolled trial with 125 patients treated with first line infliximab, and the median duration of inf- 
liximab treatment was 2.2 years. The median disease duration of discontinued infliximab group was 7.8 years. The cumulative relapse rates at 1 and 2 years were $43.9 \%$ and $52.2 \%$, respectively, which was also higher compared to the study by Song et al. The study population in the study by Song et al. had a lower rate of current smoking compared to the study by Louis et al. (7.0\% vs $39.0 \%$ ), which could affect the different relapse rates between the two studies. However, the baseline characteristics in the study by Song et al. was not better compared to the study by Louis et al. in terms of higher rates of ileum or ileo-colonic location of disease ( $70.4 \%$ vs $68 \%)$, stricturing behavior of disease ( $22.5 \%$ vs $10.0 \%)$, perianal disease (35.0\% vs $59.2 \%$ ), and previous history of intestinal surgery ( $25.4 \%$ vs $22.0 \%$ ). The retrospective real-life study design might have affected the study outcomes and reduced relapse rates.

The re-treatment with the same anti-TNF was effective in $96.1 \%$ (50/52 patients) in the study by Song et al. The poor response rate to the re-treatment was reported to be 9\% (4/46 patients) and 12\% (5/43 patients) in the previous RCTs, ${ }^{6,7}$ which could be related to the immunogenicity of the anti-TNF agent.

This study showed long-term outcomes after the discontinuation of anti-TNF agents in Korea. However, careful consideration should be required before deciding the discontinuation of anti-TNFs, because of still its high rates of relapse and possible immunogenicity. Further prospective RCTs should be warranted for the discontinuation issue of anti-TNFs. Also, predicting factors including clinical, biochemical, and molecular parameters should be determined to appropriately select patients who can discontinue the drug without future development of disease relapse.

\section{CONFLICTS OF INTEREST}

J.H.C. is an editorial board member of the journal but was not involved in the peer reviewer selection, evaluation, or decision process of this article. No other potential conflicts of interest relevant to this article were reported.
ORCID

Jihye Park https://orcid.org/0000-0002-5836-8735

Jae Hee Cheon

\section{REFERENCES}

1. Hisamatsu T, Suzuki Y, Kobayashi M, et al. Long-term safety and effectiveness of adalimumab in Japanese patients with Crohn's disease: 3-year results from a real-world study. Intest Res. Epub 2020 Nov 20. https://doi.org/10.5217/ir.2020. 00025 .

2. Ogata H, Hagiwara T, Kawaberi T, Kobayashi M, Hibi T. Safety and effectiveness of adalimumab in the treatment of ulcerative colitis: results from a large-scale, prospective, multicenter, observational study. Intest Res. Epub 2020 Nov 10. https://doi.org/10.5217/ir.2020.00033.

3. Phan H, Weideman RA, Cipher DJ, Feagins LA. Safety of tumor necrosis factor inhibitor use in patients with concomitant malignancy. Intest Res 2020;18:282-288.

4. Theodoraki E, Orfanoudaki E, Foteinogiannopoulou K, Legaki E, Gazouli M, Koutroubakis IE. Is there a correlation between infliximab trough levels and the development of adverse events in patients with inflammatory bowel disease? Intest Res. Epub 2020 Aug 18. https://doi.org/10.5217/ ir.2020.00042.

5. Song JH, Kang EA, Park SK, et al. Long-term outcomes after the discontinuation of anti-tumor necrosis factor- $\alpha$ therapy in patients with inflammatory bowel disease under clinical remission: a Korean Association for the Study of Intestinal Disease Multicenter Study. Gut Liver 2021;15:752-762.

6. Kobayashi T, Motoya S, Nakamura S, et al. Discontinuation of infliximab in patients with ulcerative colitis in remission (HAYABUSA): a multicentre, open-label, randomised controlled trial. Lancet Gastroenterol Hepatol 2021;6:429-437.

7. Louis E, Mary JY, Vernier-Massouille G, et al. Maintenance of remission among patients with Crohn's disease on antimetabolite therapy after infliximab therapy is stopped. Gastroenterology 2012;142:63-70. 